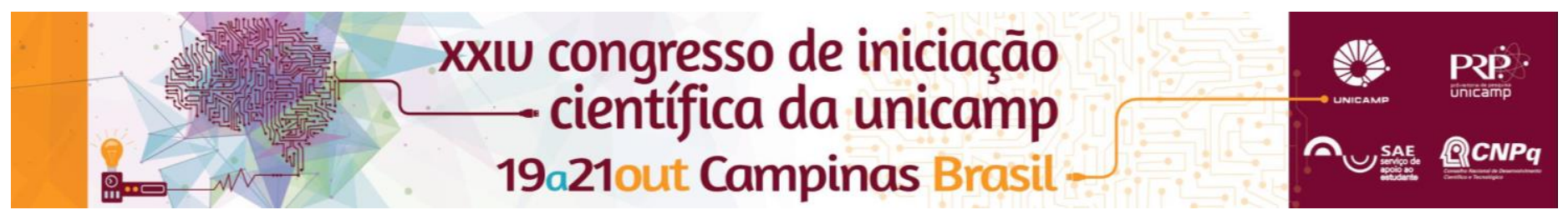

\title{
Chemical substitution effects on YMn2 intermetallic antiferromagnet
}

\author{
Denise S. Christovam, Jean C. Souza, Camilo B. R. Jesus, Mario M. Piva, Matheus Radaelli, Pascoal G. Pagliuso
}

\begin{abstract}
In this study we present the results of synthesis and characterization of single crystals of the antiferromagnetic intermetallic compound YMn2, unalloyed and with chemical substitutions, using a Sn-flux technique. In particular, we inspect the effects of $\mathrm{Cu}$ chemical substitutions in the $\mathrm{Mn}$ site of $\mathrm{YMn} 2$, and investigate the $\mathrm{Cu}$-concentration dependence of the magnetic properties of this compound. Comparing our results to previous ones, we discuss the interplay between its doping induced properties.
\end{abstract}

\section{Key words:}

Intermetallic compounds, antiferromagnetism, chemical substitution

\section{Introduction}

1This compound is a antiferromagnet with $T_{N}=100 \mathrm{~K}$ that crystallizes in a cubic C15 Laves structure, which presents significant volume change (4.6\%) near the antiferromagnetic transition, pointing to a strong magnetoelastic coupling in this material. Previous studies concerning chemical substitutions using scandium and aluminum, performed in polycrystals of $\mathrm{Y}_{1-\mathrm{x}} \mathrm{Sc}_{\mathrm{x}} \mathrm{Mn}_{2}$ and $Y\left(M n_{1-x} A I_{x}\right)_{2}$, have revealed the presence of strong magnetic fluctuations nearby the a point in the phase diagram where the Néel Temperature extrapolates to $\mathrm{T}=$ 0 . These magnetic fluctuations have spin dynamics very similar to those found in Cu-based and heavy fermion superconductors. We intend to synthesise pure and $\mathrm{Cu}$ substituted single crystals, and investigate the effects in magnetic ordering, seeking for a change in its characteristics that may lead to a new superconductor.

\section{Results and Discussion}

Through structural measurements such as energydispersive X-ray spectroscopy (EDS) and X-ray powder diffraction (XRD), we verified that in our attempts to grow pure single crystals we weren't able to obtain the desired phases due to the formation of YSn2 and MnSn2, and we are currently trying to fix this in the synthesis process. Besides, we successfully managed to substitute copper in $\mathrm{Y}\left(\mathrm{Mn}_{1-\mathrm{x}} \mathrm{Cu}_{\mathrm{x}}\right)_{2}$, with $\mathrm{x}=0.2$ and $\mathrm{x}=0.35$.

We measured the magnetic response in high fields (1 Tesla), resistance and heat capacity of the samples, and found the antiferromagnetic transition characteristic of YMn2, in addition to transitions due to MnSn2 in YMnSn5 samples (original sample we believe contains YMn2).

The data shows a possible mitigation of the ordering, as well as a shift in critical temperatures of the high temperature transition, as a function of $\mathrm{x}$. This probably means $\mathrm{Cu}$ ions are interfering with $\mathrm{Mn}$ magnetic ordering. As copper have magnetic and nonmagnetic ions it might be possible to control the way it changes the phase's ordering and introduce a new superconducting phase.

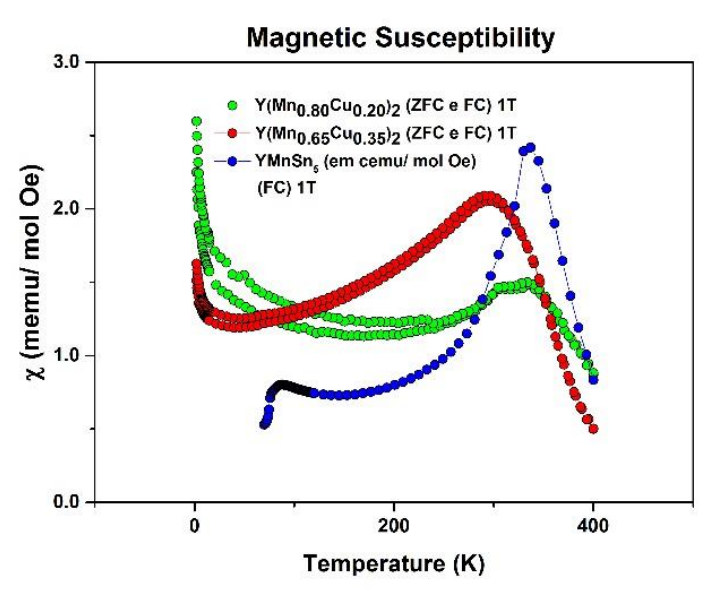

\section{Conclusions}

We managed to successfully synthesise chemically substituted the samples with copper, characterizing structurally and macroscopically (magnetic, electric and thermal response) the samples, verifying the consequences of $\mathrm{Cu}$ presence in the lattice.

\section{Acknowledgement}

I would like to thank FAPESP, Capes, CNPq and Unicamp for the financial support, and all the opportunities to introduce this study to the community.

\footnotetext{
${ }^{1}$ Nakamura, Y.; Shiga, M. and Kawano, S. Physica 120B, 1983, 202, 215.

${ }^{2}$ Wada, H., Nakamura, H.; Fukami, E.; Yoshimura, K. and Shiga, M. Journal of Magnetism and Magnetic Materials 70, 1987, 17, 19.
}

Image 1. Magnetic Susceptibility of $\mathrm{YMnSn} 5$ and $\mathrm{Y}\left(\mathrm{Mn}_{1-}\right.$ $\left.{ }_{x} \mathrm{Cu}_{\mathrm{x}}\right)_{2}(\mathrm{x}=0.2$ and $\mathrm{x}=0.35)$ 